\title{
Spin orientation and sign of the Rashba splitting in $\mathrm{Bi} / \mathrm{Cu}(111)$
}

\author{
Hendrik Bentmann, ${ }^{1}$ Takuya Kuzumaki, ${ }^{2}$ Gustav Bihlmayer, ${ }^{3}$ Stefan Blügel, ${ }^{3}$ Eugene V. Chulkov, ${ }^{4,5}$ \\ Friedrich Reinert, ${ }^{1,6}$ and Kazuyuki Sakamoto ${ }^{2}$ \\ ${ }^{1}$ Experimentelle Physik VII and Röntgen Research Center for Complex Material Systems (RCCM), Universität Würzburg Am Hubland, \\ D-97074 Würzburg, Germany \\ ${ }^{2}$ Graduate School of Advanced Integration Science, Chiba University, Chiba 263-8522, Japan \\ ${ }^{3}$ Peter Grünberg Institut and Institute for Advanced Simulation, Forschungszentrum Jülich and JARA, D-52425 Jülich, Germany \\ ${ }^{4}$ Donostia International Physics Center, 20018 San Sebastián/Donostia, Basque Country, Spain \\ ${ }^{5}$ Departamento de Física de Materiales and Centro Mixto CSIC-UPV/EHU, UPV/EHU, Apartado 1072, 20080 San Sebastián/Donostia, \\ Basque Country, Spain \\ ${ }^{6}$ Karlsruher Institut für Technologie (KIT), Gemeinschaftslabor für Nanoanalytik,, D-76021 Karlsruhe, Germany
}

(Received 5 July 2011; published 19 September 2011)

\begin{abstract}
Whereas the magnitude of the Rashba spin splitting at surfaces has been studied in detail, less is known about its sign which determines the precise spin orientation of the electronic states. We investigate the microscopic origin of this sign by spin-resolved photoemission experiments and first-principles calculations on the strongly spin-orbit coupled surface states in $\mathrm{Bi} / \mathrm{Cu}(111)$. We conclude that the sign of the Rashba splitting is determined by the particular charge asymmetry near the atomic cores. The precise spin orientation on heavy-element surfaces thus can comprise information on wave-function localizations and related aspects otherwise hardly accessible by other experimental methods.
\end{abstract}

DOI: 10.1103/PhysRevB.84.115426

PACS number(s): 73.20.At, 71.70.Ej, 79.60.-i

\section{INTRODUCTION}

In a solid-state system that preserves both time-reversal and spatial inversion symmetry the electronic states are necessarily spin degenerate. ${ }^{1}$ This degeneracy is broken by the spinorbit interaction for electrons moving in two-dimensional (2D) surface-, interface- or quantum-well geometries which give rise to inversion-asymmetric confinement potentials., ${ }^{2,3}$ This Rashba effect is an important mechanism in the field of spintronics as it can be utilized for the manipulation of spin-polarized currents in designated semiconductor heterojunctions. ${ }^{4,5}$ Particularly large Rashba splittings are observed in the electronic structure of clean or monolayer covered heavy-element surfaces. ${ }^{6-12}$ Related spin-splitting mechanisms are found for the surface states on topological insulators. ${ }^{13,14}$

The Rashba effect in a 2D electron gas leads to a splitting of the free-electron dispersion via a potential gradient perpendicular to the plane of confinement,

$$
E_{ \pm}(\boldsymbol{k})=E_{0}+\frac{\hbar^{2} \boldsymbol{k}^{2}}{2 m^{*}} \pm|\alpha||\boldsymbol{k}|,
$$

where $m^{*}$ is the effective mass and the absolute value of the Rashba parameter $\alpha$ measures the size of the spin splitting [see Fig. 1(a)]. The spin orientation of the states $E_{ \pm}$is then given by

$$
\boldsymbol{P}_{ \pm}(\boldsymbol{k})= \pm \frac{\alpha}{|\alpha|}\left(-k_{y}, k_{x}, 0\right) /|\boldsymbol{k}|
$$

In Eq. (2) we use the same sign convention as in Ref. 15. The spin polarization vector $\boldsymbol{P}_{ \pm}$is oriented in plane and perpendicular to the wave vector. Furthermore, the branches $E_{ \pm}$have opposite spin orientations $\boldsymbol{P}_{+}=-\boldsymbol{P}_{-}$. The absolute directions of $\boldsymbol{P}_{ \pm}$are determined by the sign of the Rashba parameter $\alpha{ }^{15,16}$ Note that the sign of the effective mass defines whether the branch $E_{+}$(and accordingly $E_{-}$) is the outer or the inner branch in Fig. 1. As a result the spin orientation of the inner and of the outer branch depend on both the sign of the Rashba parameter and the sign of the effective mass [see Fig. 1(b)].

Previous experimental and theoretical work gave increasingly detailed insights into parameters that determine the magnitude of the Rashba parameter in surface and thin-film systems. ${ }^{6,11,17-29}$ In the present paper we address a related but less explored issue, namely, the sign of the Rashba parameter at surfaces. At first glance one may not expect the sign of $\alpha$ to vary between different surfaces as the potential gradient between crystal and vacuum probed by the surface-state wave function should be similar for all systems. On the other hand, Rashba-split quantum-well states in $\mathrm{Pb} / \mathrm{Si}(111)$ show a spin
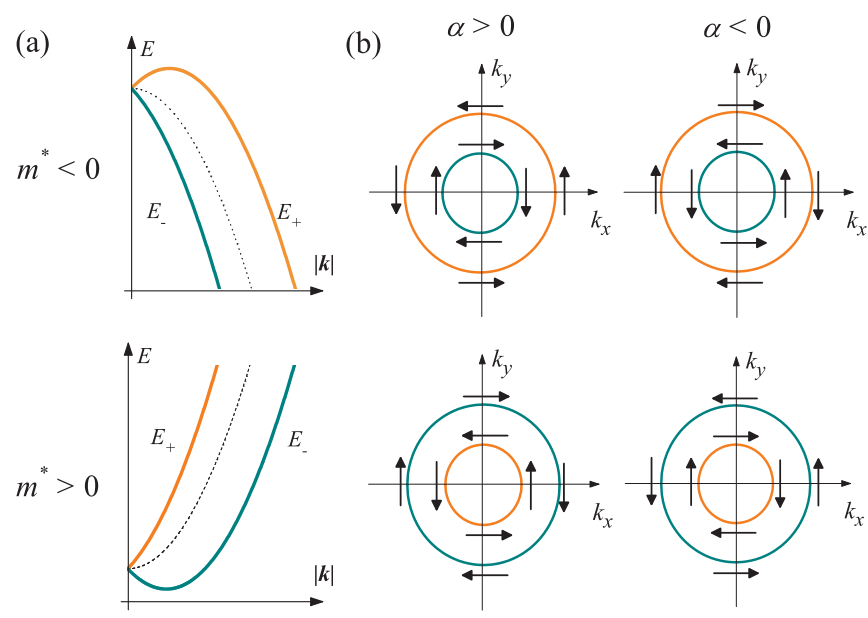

FIG. 1. (Color online) Dispersion in (a) and spin orientation in (b) for the Rashba model in a two-dimensional electron gas: The spin-orbit interaction splits the free-electron parabola (dashed line) into the branches $E_{ \pm}$. The spin orientation, indicated by arrows, of the inner and outer branch depends on the sign of the Rashba parameter $\alpha$ and of the effective mass $m^{*}$. 
orientation that is reversed when compared to the Shockleytype surface state on $\mathrm{Au}(111))^{30}$ This has been attributed to competing effects at the two terminating interfaces of the $\mathrm{Pb}$ film.

In this paper we investigate the sign of the Rashba splitting at surfaces and its microscopic origin using spin- and angleresolved photoelectron spectroscopy (SARPES) for the surface alloy $\mathrm{Bi} / \mathrm{Cu}(111)$. The measured spin orientation in $\mathrm{Bi} / \mathrm{Cu}(111)$ is in accordance with Eq. (2) and implies a negative Rashba parameter $\alpha$. We confirm these findings by a first-principles calculation of the spin orientation. Employing a simple model in combination with our calculations, we argue that the sign of $\alpha$ is determined by local wave-function asymmetries in the vicinity of the Bi nuclei. Thus, more generally, our study identifies the sign of the Rashba parameter at surfaces as an important experimental observable. In particular, when combined with calculations, it can provide information on wave-function localization, orbital character, and symmetries of electronic states. We compare our results for $\mathrm{Bi} / \mathrm{Cu}(111)$ with previous investigations on $\mathrm{Au}(111) .{ }^{15}$

Noteworthy, the spin-split surface states of topological insulators show related spin orientations as depicted in Fig. 1(b). ${ }^{31}$ It is conceivable that similar mechanisms as described here for Rashba-split surface states also have an impact on the precise spin orientation of these topological states and hence on the sign of spin currents carried by them.

The $\mathrm{Bi} / \mathrm{Cu}(111)$ surface alloy forms a Rashba-split surface state with negative effective mass and mainly $\mathrm{Bi} s p_{z}$ orbital character. ${ }^{27,28,32}$ Similar to $\mathrm{Au}(111)$, this $s p_{z}$ state shows a dispersion perfectly matching the one prescribed by Eq. (1): two parabolic bands shifted in $k$ and circular momentum distributions (see Ref. 28 for further details). In addition, another surface state of $p_{x} p_{y}$ orbital character has been found for $\mathrm{Bi} / \mathrm{Cu}(111)$. In this paper we will focus on the Rashba splitting and spin orientation of the $s p_{z}$ surface state.

\section{EXPERIMENTAL AND THEORETICAL DETAILS}

Spin-resolved and spin-integrated experiments were carried out at room temperature (RT) using a newly designed SARPES setup at Chiba University, Japan. The spectrometer consists of a Scienta R4000 electron analyzer for energy and angular resolution as well as a Scienta Mott detector operated at $25 \mathrm{keV}$ for the spin analysis. The geometry of the setup allows for a parallel detection of two components of the spin orientation, in this case the component normal to the surface and the in-plane component perpendicular to the wave vector (henceforth the Rashba direction). We employed a monochromated Xe discharge lamp (MB Scientific) as well as a nonmonochromated He lamp as UV-light sources. For all presented measurements we used excitation energies of $8.44 \mathrm{eV}$ (Xe I) or $21.22 \mathrm{eV}$ ( $\mathrm{He} \mathrm{I})$. We conducted the experiments at energy resolutions of $\sim 50 \mathrm{meV}$ for the ARPES and $100-160 \mathrm{meV}$ for the SARPES measurements. The SAPRES spectra were taken with an acceptance angle of $\pm 3^{\circ}$ for Xe I and $\pm 1.5^{\circ}$ for He I. The effective Sherman function for the SARPES experiments was 0.18. Careful in situ preparation of the single-crystalline $\mathrm{Cu}(111)$ substrate by repeated cycles of Ar-ion sputtering and annealing to $\sim 1000 \mathrm{~K}$ resulted in a clean and well-ordered surface as verified by
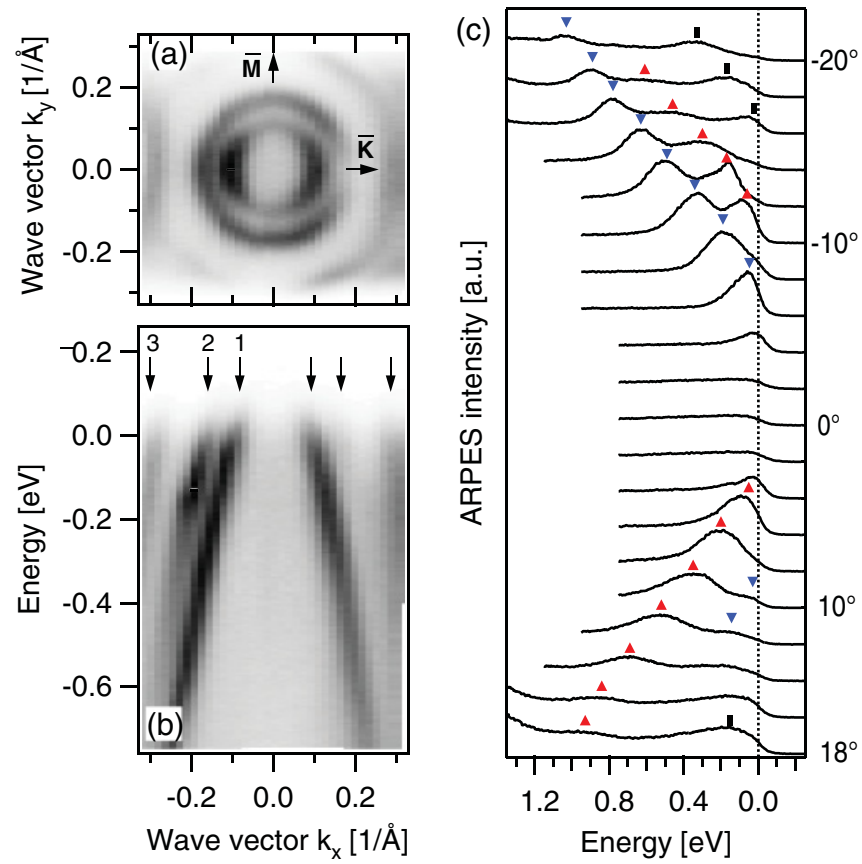

FIG. 2. (Color online) Spin-integrated photoemission from $\mathrm{Bi} / \mathrm{Cu}(111)$ obtained with Xe I excitation. (a) and (b) show the Fermi surface and the band structure along the $\bar{\Gamma} \bar{K}$ direction. In (c) we display energy distribution curves around $\bar{\Gamma}$ obtained from the same data set as the map in (b).

the photoemission linewidth of the $L$-gap surface state. ${ }^{33}$ The surface alloy reconstruction $\mathrm{Bi}-\mathrm{Cu}(111)(\sqrt{3} \times \sqrt{3}) R 30^{\circ}$ was obtained after evaporation of 1-2 monolayers (ML) of Bi and subsequent postannealing at $\sim 500 \mathrm{~K}$. We verified the qualtity of the surface by low-energy electron diffraction (LEED) and ARPES. The base pressure for all experiments was lower than $2 \times 10^{-10}$ mbar.

The calculation of the surface electronic structure is based on the full-potential linearized augmented plane-wave method and density functional theory as implemented in the FLEUR code. ${ }^{34}$ The surface was simulated in a slab geometry with a ten-layer $\mathrm{Cu}(111)$ film terminated on one end by the $\mathrm{Bi}-\mathrm{Cu}(111)(\sqrt{3} \times \sqrt{3}) R 30^{\circ}$ surface alloy reconstruction. For further specifications of the calculation, we refer the reader to Ref. 28.

\section{SPIN ORIENTATION IN Bi/Cu(111)}

We first briefly discuss the spin-integrated electronic structure of $\mathrm{Bi} / \mathrm{Cu}(111)$ as obtained with Xe I excitation energy at RT (see Fig. 2). The band structure along $\bar{\Gamma} \bar{K}$ and the Fermi surface (FS) reproduce our earlier results in Ref. 28. The FS consists of one hexagonal and two circular contours. Three states labeled in Fig. 2(b) by ascending numbers cross the Fermi energy at $\pm 0.1 \AA^{-1}, \pm 0.17 \AA^{-1}$, and $\pm 0.29 \AA^{-1}$. We identify the features " 1 " and " 2 " with the inner and the outer branch of the $s p_{z}$ surface state. Branch " 3 " corresponds to the $p_{x} p_{y}$ state. Note that, despite temperature-induced spectral broadening, the three bands are still well resolved at RT. This is particularly evident from Fig. 2(c), where we show energy distribution curves (EDCs) for emission angles 

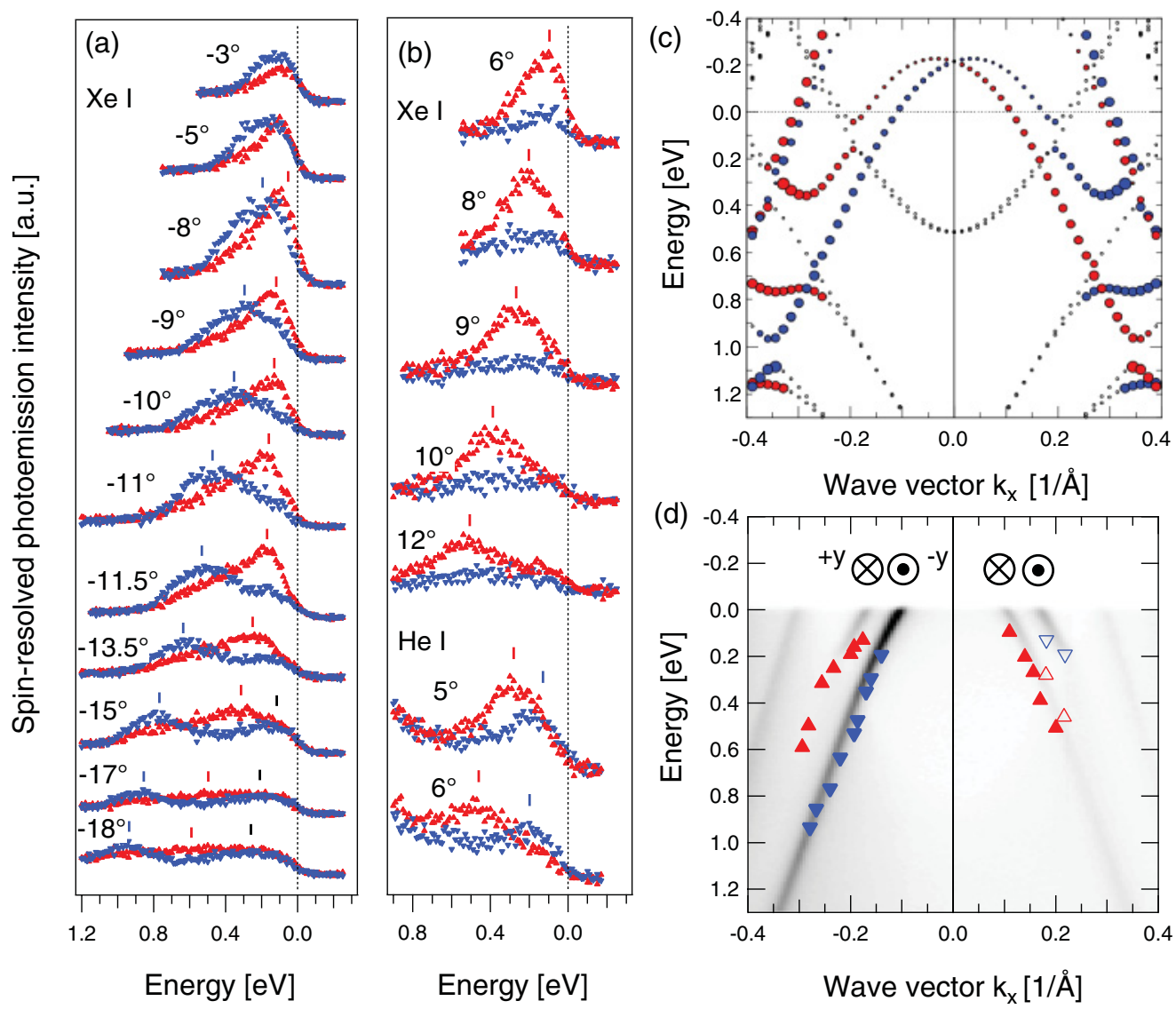

FIG. 3. (Color online) Spin-resolved electronic structure of $\mathrm{Bi} / \mathrm{Cu}(111)$. In (a) and (b) we show spin-resolved energy distribution curves along $\bar{\Gamma} \bar{K}$ for negative and positive emission angles. The respective excitation energies are indicated. The spectra in (a) were recorded with an energy resolution of $160 \mathrm{meV}$, and the ones in (b) with $160 \mathrm{meV}$ (He I) and $100 \mathrm{meV}$ (Xe I). In (c) we present a first-principles calculation of the spin-resolved surface electronic structure. (d) shows a high-resolution photoemission map of Bi/Cu(111) (taken from Ref. 28). Additional markers indicate the peak positions obtained from the spin-resolved datasets in (a) and (b). For all panels the spin-quantization axis is in plane and perpendicular to the wave vector $k_{x}(\bar{\Gamma} \bar{K})$. Red (light) and blue (dark) symbols correspond to a spin orientation parallel to $y$ and $-y$, respectively. In (d) closed (open) symbols indicate data points obtained with Xe I (He I) excitation.

$\theta_{e}$ around the surface normal in steps of $2^{\circ}$ (at the Fermi level $\theta_{e}=20^{\circ}$ corresponds to $k_{x}=0.35 \AA^{-1}$ for Xe I). The peak positions are indicated where red (upward pointing) and blue (downward pointing) markers refer to the anticipated opposite spin orientations of the $s p_{z}$ branches imposed by the Rashba model. Black (rectangular) markers denote the $p_{x} p_{y}$ branch. Note that while the inner $s p_{z}$ branch shows a fairly symmetric intensity behavior around $\bar{\Gamma}$, the spectral weight of the outer $s p_{z}$ state is almost entirely suppressed for positive $\theta_{e}$. This is a result of strongly varying photoemission matrix elements which have been observed for other surface alloys in a similar fashion. ${ }^{26}$

Having confirmed the results of previous studies on the spin-integrated electronic structure, we next present our experimental findings on the spin orientation in $\mathrm{Bi} / \mathrm{Cu}(111)$. In Fig. 3 we show spin-resolved EDCs $N_{\uparrow}$ (red upward triangles) and $N_{\downarrow}$ (blue downward triangles) along $\bar{\Gamma} \bar{K}\left(k_{x}\right)$ for negative emission angles in Fig. 3(a) and for positive angles in Fig. 3(b). The spectra were recorded with Xe I excitation except for two spectra which were taken by He I excitation [see according to the indications in Figs. 3(a) and 3(b)]. Both panels refer to a spin quantization axis parallel to the $y$ axis (Rashba direction).
Spin up $(\uparrow)$ and spin down $(\downarrow)$ correspond to a spin orientation pointing in the $y$ and $-y$ directions, respectively. Note that we use a right-handed coordinate system with the $z$ axis pointing out of the surface plane toward the vacuum. Considering the EDCs in Fig. 3(a) for $\left|\theta_{e}\right| \geqslant 8^{\circ}$ we find peaks in $N_{\uparrow}$ and $N_{\downarrow}$ which are separated in energy. Both of them disperse to higher binding energies for increasing $\left|\theta_{e}\right|$. By comparison with the spin-integrated data in Fig. 2 we can associate these peaks with the inner and the outer $s p_{z}$ branch. Hence, we find that these bands are spin polarized with opposite spin orientations along the Rashba direction. In the EDCs taken with Xe I for positive $\theta_{e}$ in Fig. 3(b) we observe a dispersive feature only for $N_{\uparrow}$ which is ascribed to the inner $s p_{z}$ branch. We thus conclude that the inner $s p_{z}$ state reverses its spin orientation for opposite $k$ directions. The absence of a second peak is attributed to the aforementioned matrix element suppression of the outer branch. In order to avoid the suppression of the outer $s p_{z}$ branch, we collected additional data using $\mathrm{He}$ I excitation. Note that equivalent wave vectors correspond to approximately half the emission angle for He I compared to Xe I. Indeed, the spectra in for He I excitation show an additional peak in $N_{\downarrow}$ at lower binding energies which is attributed to 
the outer $s p_{z}$ state. This confirms that the spin orientation of the outer $s p_{z}$ branch is reversed for opposite $k$ directions as well. In our measurements of the out-of-plane component of the spin orientation (not shown) we did not find a significant spin polarization and it is hence estimated to be smaller than $\sim 5 \%$.

The experimental results on the spin-resolved electronic structure of $\mathrm{Bi} / \mathrm{Cu}(111)$ are summarized in Fig. 3(d). We plot a spin-integrated high-resolution ARPES map obtained with He I (taken from Ref. 28) and, additionally, the peak positions obtained from the spin-resolved EDCs in Figs. 3(a) and 3(b). Again, red (upward) and blue (downward) symbols represent a spin orientation in the $y$ and $-y$ directions, respectively. Both datasets show a sound agreement especially for the inner state whereas the remaining discrepancies are attributed to the considerably reduced experimental resolution in the spin-resolved measurements. To conclude, we find a spin orientation of the $s p_{z}$ surface state according to Eq. (2) as prescribed by the Rashba model. The absolute directions match those depicted in Fig. 1(b) for the two combinations $\left(\alpha>0, m^{*}>0\right)$ and $\left(\alpha<0, m^{*}<0\right)$. Thus, given the negative effective mass of the band dispersion, the experimentally determined Rashba parameter $\alpha$ for $\mathrm{Bi} / \mathrm{Cu}(111)$ is negative.

To further corroborate the experimental findings, we consider our first-principles calculation of the spin-polarized surface band structure [see Fig. 3(c)]. Comparing Figs. 3(d) and 3(c) we infer that the calculated surface band structure quantitatively reproduces the experimentally observed dispersion. More importantly, for the purpose of the present study, also the calculated spin orientations of the individual branches agree with our experimental results and yield a negative $\alpha$.

\section{SIGN OF THE RASHBA PARAMETER}

Based on our experimental and theoretical results, we will now discuss the origin of the sign of the Rashba parameter and the corresponding spin orientation. Previous investigations on the basis of first-principles calculations revealed a markedly local character of the Rashba effect at surfaces. ${ }^{19}$ The splitting size is determined within $\sim 0.2 \AA$ around the nucleus where the atomic field gradients are largest. Reflecting the broken structural inversion symmetry, surface states can exhibit a considerably asymmetric charge distribution around the atomic nuclei. In fact, such an asymmetry is a necessary condition for a spin splitting to occur at all. Its precise form has been shown to strongly influence the magnitude of $\alpha{ }^{19,25,35}$ Hence, it is conceivable that also the sign of $\alpha$ is determined by details of the local wave-function asymmetry in the vicinity of the nuclei.

To elaborate on this point, we adopt the simple expression for the Rashba parameter $\alpha=2 / c^{2} \int \phi^{2}(z) \partial_{z} V d^{3} \boldsymbol{r}$, suggested in Ref. 25, assuming a free-electron behavior in the surface plane and a confined wave function $\phi(z)$ perpendicular to the plane. Note that for a strictly $2 \mathrm{D}$ case $\left[\phi^{2}(z)=\delta\left(z-z_{0}\right)\right.$, with the Dirac-Delta function $\delta(z)]$ the above expression yields the same definition for $\alpha$ as the one used in Ref. 15, which then leads to the sign convention in Eq. (2). Close to the atomic cores the potential $V$ can be approximated by the bare Coulomb term, and hence $\partial_{z} V$ is an antisymmetric function with respect to the nucleus at $z=0$. Thus, from the above model, we would expect a sign change of $\alpha$ depending on whether an excess charge is localized on the vacuum $\left(z_{0}>0\right)$ or on the substrate $\left(z_{0}<0\right)$ facing side of the nucleus. Keeping this in mind, we next consider the calculated partial charge density of the $s p_{z}$ surface state at $\bar{\Gamma}$ (see Fig. 4). Figure 4(a) shows a charge line profile along the $z$ direction and Fig. 4(b) displays a contour plot of the charge density in the [110] plane. As anticipated, we find a clear asymmetry of the charge profile along the $z$ direction. More precisely, we notice an imbalance of the partial charge close to the $\mathrm{Bi}$ nucleus in favor of the substrate facing side $\left(z_{0}<0\right)$. For this case we would expect a negative $\alpha$, which is indeed what is found by our experiments and calculations. Hence, taken collectively, our results suggest that the negative sign of the Rashba parameter in $\mathrm{Bi} / \mathrm{Cu}(111)$ is related to the particular imbalance in the charge distribution around the $\mathrm{Bi}$ cores.

It is instructive to compare the present results for $\mathrm{Bi} / \mathrm{Cu}(111)$ with the Rashba-split surface state of $\mathrm{Au}(111)$. A
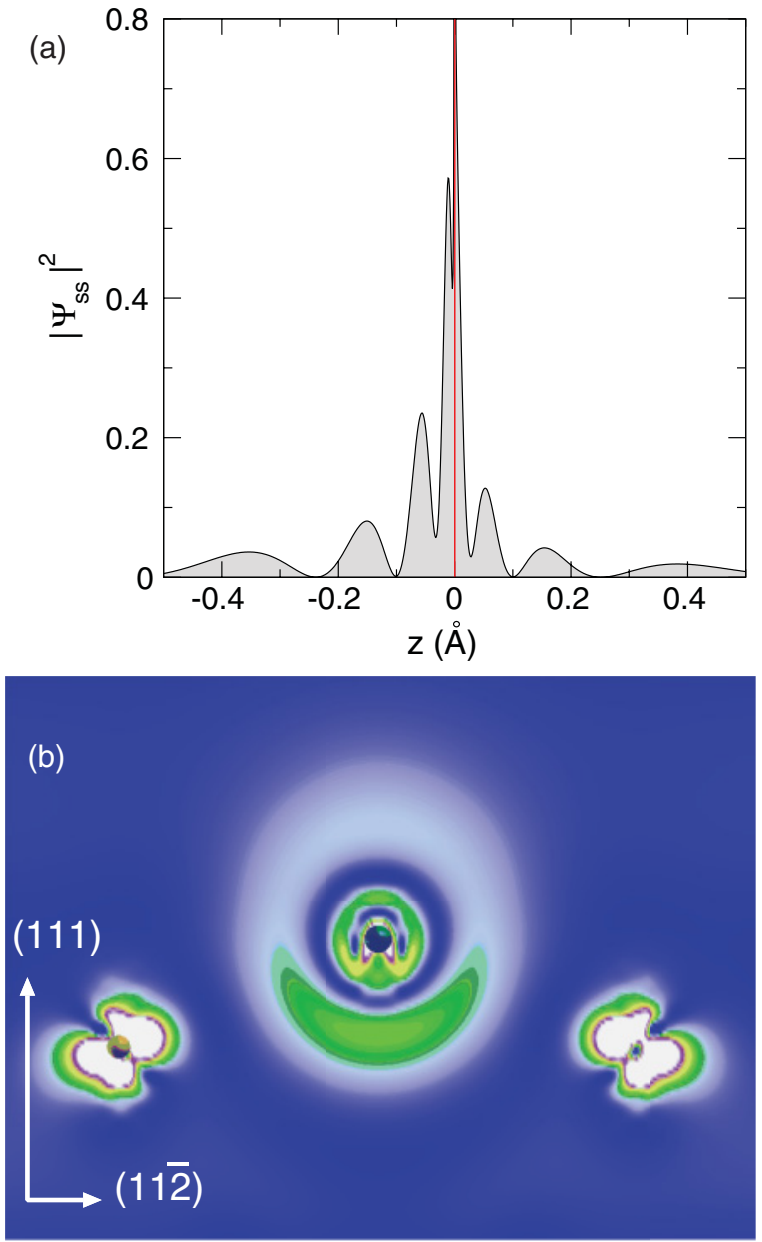

FIG. 4. (Color online) First-principles calculation of the partial charge density of the $s p_{z}$ state on $\mathrm{Bi} / \mathrm{Cu}(111)$ at the $\bar{\Gamma}$ point. (a) One-dimensional charge density profile along the $z$ direction [(111) direction]. The $\mathrm{Bi}$ atom is located at $z=0$ and the vacuum side corresponds to positive $z$ values. The charge was averaged in the $x y$ plane within an interval of $\pm 0.07 \AA$ around the nucleus. (b) Two-dimensional cut through the charge density in the [110] plane. One $\mathrm{Bi}$ atom in the center and two $\mathrm{Cu}$ atoms are indicated. 
previous experimental and theoretical study on $\mathrm{Au}(111)$ found a positive Rashba parameter. ${ }^{15}$ This result is nicely in line with our findings here because, contrary to $\mathrm{Bi} / \mathrm{Cu}(111)$, the $\mathrm{Au}(111)$ surface-state wave function is localized predominantly on the vacuum facing side of the outermost Au layer and hence the sign of $\alpha$ is changed. ${ }^{15,25}$ Note, however, that for $\mathrm{Au}(111)$ with $\left(\alpha>0, m^{*}>0\right)$ the actual spin orientations of the outer and of the inner branch are the same as for $\mathrm{Bi} / \mathrm{Cu}(111)$ with $\left(\alpha<0, m^{*}<0\right)$ (compare Fig. 1).

The particular wave-function localization for $\mathrm{Bi} / \mathrm{Cu}(111)$ discussed above can be interpreted as a result of hybridization of the adsorbate $s p_{z}$ orbitals with the underlying substrate states. This hybridization is directly inferred from Fig. 4(b), showing high partial charge at the $\mathrm{Bi}$ atom but also at the $\mathrm{Cu}$ atoms. The $s p_{z}$ surface state is thus involved in the adsorbate-substrate bonding and consequently strongly localized between the two. We expect a related behavior for the isostructural surface alloys $\mathrm{Pb} / \mathrm{Ag}(111)$ and $\mathrm{Bi} / \mathrm{Ag}(111)$ which feature analogous Rashba-split surface states. ${ }^{11,21,36}$ Our calculations show indeed that the Rashba parameter for these two systems is negative as for $\mathrm{Bi} / \mathrm{Cu}(111)$, reflecting a similar wave-function localization predominantly on the substrate side of the adsorbate atoms. These considerations exemplify that the sign of the Rashba splitting certainly bears information on bonding properties and charge localization in heavy-element surface and thin-film systems. The findings are easily generalized to a broader range of systems such as, for example, monolayer covered semiconductor surfaces with large spin splittings (see Refs. 12, 24, and 37-39).

Another interesting aspect where knowledge of the sign of the Rashba parameter may give additional insights concerns the sensitivity of surface states toward adatom adsorbtion. To illustrate this we compare previous results on the effect of Xe adsorption on the surface electronic structure of $\mathrm{Au}(111)$ and $\mathrm{Bi} / \mathrm{Ag}(111)$. For $\mathrm{Au}(111)$ a large change in binding energy of $150 \mathrm{meV}$ after adsorption of a closed Xe layer has been observed and attributed to the direct overlap of surface-state and adsorbate wave functions. ${ }^{40}$ On the other hand, the surface state of $\mathrm{Bi} / \mathrm{Ag}(111)$ is only weakly influenced by a
Xe overlayer, suggesting a considerably smaller overlap. ${ }^{41}$ At least partly these observations can be traced back to the different wave-function localization of the two surface states which is encoded in the sign of their Rashba parameter: The surface state on $\mathrm{Au}(111)$ features a higher partial charge on the vacuum side of the first layer than $\mathrm{Bi} / \mathrm{Ag}(111)$, which results in a stronger interaction with adsorbates.

\section{SUMMARY}

We have shown that the sign of the Rashba splitting in surface and thin-film systems is determined by the precise charge distribution asymmetry of a surface state close to the atomic nuclei. Thus, the sign of the Rashba parameter contains information on the real space localization of surface states which can provide additional insights in adsorbate-substrate interactions and related mechanisms. Specifically, we find a negative Rashba parameter for the surface alloy $\mathrm{Bi} / \mathrm{Cu}(111)$. This result is explained by the particular wave-function localization of this surface state which is involved in the bonding between the $\mathrm{Bi}$ adsorbate atoms and the $\mathrm{Cu}(111)$ substrate. It would be desirable to extend the present findings to more complex systems, such as states with out-of-plane or other unconventional spin orientations beyond the Rashba model (see Refs. 32, 36, and 37) as well as the surface states on topological insulators (see, e.g., Ref. 42).

\section{ACKNOWLEDGMENTS}

We gratefully acknowledge experimental support by Ryusei Tateishi and Yuta Yamamoto. G.B. would like to thank Hugo Dil for helpful discussions. This work was supported by the Grant-in-Aid for Scientific Research (A) 20244045, the G-COE programs (G-03), the Bundesministerium für Bildung und Forschung (Grant Nos. 05K10WW1/2 and 05KS1WMB/1), and the Deutsche Forschunsgsgemeinschaft (FOR 1162). H.B. acknowledges support by the Japan Society for the Promotion of Science.

\footnotetext{
${ }^{1}$ C. Kittel, Quantum Theory of Solids (Wiley, New York, 1963).

${ }^{2} \mathrm{R}$. Winkler, Spin-Orbit Coupling Effects in Two-Dimensional Electron and Hole Systems, Springer Tracts in Modern Physics Vol. 191 (Springer, Berlin, 2003).

${ }^{3}$ Y. A. Bychkov and E. I. Rashba, JETP Lett. 39, 78 (1984).

${ }^{4}$ S. Datta and B. Das, Appl. Phys. Lett. 56, 665 (1990).

${ }^{5}$ H. C. Koo, J. H. Kwon, J. Eom, J. Chang, S. H. Han, and M. Johnson, Science 325, 1515 (2009).

${ }^{6}$ S. LaShell, B. A. McDougall, and E. Jensen, Phys. Rev. Lett. 77, 3419 (1996).

${ }^{7}$ Y. M. Koroteev, G. Bihlmayer, J. E. Gayone, E. V. Chulkov, S. Blügel, P. M. Echenique, and P. Hofmann, Phys. Rev. Lett. 93, 046403 (2004).

${ }^{8}$ D. Pacilé, C. R. Ast, M. Papagno, C. Da Silva, L. Moreschini, M. Falub, A. P. Seitsonen, and M. Grioni, Phys. Rev. B 73, 245429 (2006).
}

${ }^{9}$ T. Hirahara, T. Nagao, I. Matsuda, G. Bihlmayer, E. V. Chulkov, Y. M. Koroteev, P. M. Echenique, M. Saito, and S. Hasegawa, Phys. Rev. Lett. 97, 146803 (2006).

${ }^{10}$ I. Barke, F. Zheng, T. K. Rügheimer, and F. J. Himpsel, Phys. Rev. Lett. 97, 226405 (2006).

${ }^{11}$ C. R. Ast, J. Henk, A. Ernst, L. Moreschini, M. C. Falub, D. Pacilé, P. Bruno, K. Kern, and M. Grioni, Phys. Rev. Lett. 98, 186807 (2007).

${ }^{12}$ K. Sakamoto, H. Kakuta, K. Sugawara, K. Miyamoto, A. Kimura, T. Kuzumaki, N. Ueno, E. Annese, J. Fujii, A. Kodama et al., Phys. Rev. Lett. 103, 156801 (2009).

${ }^{13}$ D. Hsieh, Y. Xia, L. Wray, D. Qian, A. Pal, J. H. Dil, J. Osterwalder, F. Meier, G. Bihlmayer, C. L. Kane et al., Science 323, 919 (2009).

${ }^{14}$ D. Hsieh, Y. Xia, D. Qian, L. Wray, J. H. Dil, F. Meier, J. Osterwalder, L. Patthey, J. G. Checkelsky, N. P. Ong et al., Nature (London) 460, 1101 (2009). 
${ }^{15}$ J. Henk, M. Hoesch, J. Osterwalder, A. Ernst, and P. Bruno, J. Phys. Condens. Matter 16, 7581 (2004).

${ }^{16}$ J. Henk, A. Ernst, and P. Bruno, Phys. Rev. B 68, 165416 (2003).

${ }^{17}$ L. Petersen and P. Hedegåard, Surf. Sci. 459, 49 (2000).

${ }^{18}$ F. Reinert, J. Phys. Condens. Matter 15, S693 (2003).

${ }^{19}$ G. Bihlmayer, Y. M. Koroteev, P. M. Echenique, E. V. Chulkov, and S. Blügel, Surf. Sci. 600, 3888 (2006).

${ }^{20}$ J. Premper, M. Trautmann, J. Henk, and P. Bruno, Phys. Rev. B 76, 073310 (2007).

${ }^{21}$ G. Bihlmayer, S. Blügel, and E. V. Chulkov, Phys. Rev. B 75, 195414 (2007).

${ }^{22}$ A. M. Shikin, A. Varykhalov, G. V. Prudnikova, D. Usachev, V. K. Adamchuk, Y. Yamada, J. Riley, and O. Rader, Phys. Rev. Lett. 100, 057601 (2008).

${ }^{23}$ C. R. Ast, G. Wittich, P. Wahl, R. Vogelgesang, D. Pacilé, M. C. Falub, L. Moreschini, M. Papagno, M. Grioni, and K. Kern, Phys. Rev. B 75, 201401 (2007).

${ }^{24}$ S. Hatta, T. Aruga, Y. Ohtsubo, and H. Okuyama, Phys. Rev. B 80, 113309 (2009).

${ }^{25}$ M. Nagano, A. Kodama, T. Shishidou, and T. Oguchi, J. Phys. Condens. Matter 21, 064239 (2009).

${ }^{26}$ L. Moreschini, A. Bendounan, I. Gierz, C. R. Ast, H. Mirhosseini, H. Höchst, K. Kern, J. Henk, A. Ernst, S. Ostanin et al., Phys. Rev. B 79, 075424 (2009).

${ }^{27}$ L. Moreschini, A. Bendounan, H. Bentmann, M. Assig, K. Kern, F. Reinert, J. Henk, C. R. Ast, and M. Grioni, Phys. Rev. B 80, 035438 (2009).

${ }^{28}$ H. Bentmann, F. Forster, G. Bihlmayer, E. V. Chulkov, L. Moreschini, M. Grioni, and F. Reinert, Europhys. Lett. 87, 37003 (2009).
${ }^{29}$ A. G. Rybkin, A. M. Shikin, V. K. Adamchuk, D. Marchenko, C. Biswas, A. Varykhalov, and O. Rader, Phys. Rev. B 82, 233403 (2010).

${ }^{30}$ J. H. Dil, F. Meier, J. Lobo-Checa, L. Patthey, G. Bihlmayer, and J. Osterwalder, Phys. Rev. Lett. 101, 266802 (2008).

${ }^{31}$ D. Hsieh, Y. Xia, L. Wray, D. Qian, A. Pal, J. H. Dil, J. Osterwalder, F. Meier, G. Bihlmayer, C. L. Kane et al., Science 323, 919 (2009).

${ }^{32}$ H. Mirhosseini, J. Henk, A. Ernst, S. Ostanin, C. T. Chiang, P. Yu, A. Winkelmann, and J. Kirschner, Phys. Rev. B 79, 245428 (2009).

${ }^{33}$ F. Reinert, G. Nicolay, S. Schmidt, D. Ehm, and S. Hüfner, Phys. Rev. B 63, 115415 (2001).

${ }^{34}$ For program description see [http://www.flapw.de].

${ }^{35}$ S. Abdelouahed and J. Henk, Phys. Rev. B 82, 193411 (2010).

${ }^{36}$ F. Meier, H. Dil, J. Lobo-Checa, L. Patthey, and J. Osterwalder, Phys. Rev. B 77, 165431 (2008).

${ }^{37}$ K. Sakamoto, T. Oda, A. Kimura, K. Miyamoto, M. Tsujikawa, A. Imai, N. Ueno, H. Namatame, M. Taniguchi, P. E. J. Eriksson et al., Phys. Rev. Lett. 102, 096805 (2009).

${ }^{38}$ I. Gierz, T. Suzuki, E. Frantzeskakis, S. Pons, S. Ostanin, A. Ernst, J. Henk, M. Grioni, K. Kern, and C. R. Ast, Phys. Rev. Lett. 103, 046803 (2009).

${ }^{39}$ K. Yaji, Y. Ohtsubo, S. Hatta, H. Okuyama, K. Miyamoto, T. Okuda, A. Kimura, H. Namatame, M. Taniguchi, and T. Aruga, Nat. Commun. 1, 17 (2010).

${ }^{40}$ F. Forster, A. Bendounan, J. Ziroff, and F. Reinert, Surf. Sci. 600, 3870 (2006).

${ }^{41}$ L. Moreschini, A. Bendounan, C. R. Ast, F. Reinert, M. Falub, and M. Grioni, Phys. Rev. B 77, 115407 (2008).

${ }^{42}$ Y. Xia, D. Qian, D. Hsieh, L. Wray, A. Pal, H. Lin, A. Bansil, D. Grauer, Y. S. Hor, R. J. Cava et al., Nat. Phys. 5, 398 (2009). 